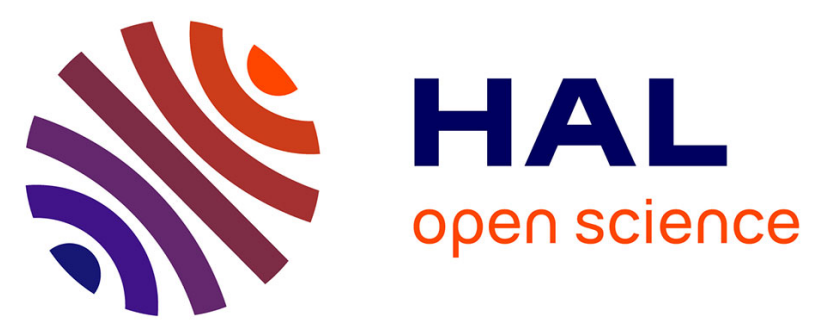

\title{
A Nonlinear Model for Nano-Electro Mechanical Mass Sensing Signals Processing
}

Thomas Fortin, Bogdan Vysotskyi, Martial Defoort, Adrien Reynaud, Szu-Hsueh Lai, Sergio Dominguez-Medina, Kavya Clement, Vaitson Çumaku, Sébastien Hentz, Christophe D. Masselon

\section{To cite this version:}

Thomas Fortin, Bogdan Vysotskyi, Martial Defoort, Adrien Reynaud, Szu-Hsueh Lai, et al.. A Nonlinear Model for Nano-Electro Mechanical Mass Sensing Signals Processing. IEEE Sensors Journal, 2021，10.1109/JSEN.2021.3103713 . hal-03455517

\section{HAL Id: hal-03455517 https://hal.science/hal-03455517}

Submitted on 13 Dec 2021

HAL is a multi-disciplinary open access archive for the deposit and dissemination of scientific research documents, whether they are published or not. The documents may come from teaching and research institutions in France or abroad, or from public or private research centers.
L'archive ouverte pluridisciplinaire HAL, est destinée au dépôt et à la diffusion de documents scientifiques de niveau recherche, publiés ou non, émanant des établissements d'enseignement et de recherche français ou étrangers, des laboratoires publics ou privés.

\section{(ㅇ)(1) $\$$}

Distributed under a Creative Commons Attribution - NonCommerciall 4.0 International 


\section{IEEE Copyright Notice}

(C) 2021 IEEE. Personal use of this material is permitted. Permission from IEEE must be obtained for all other uses, in any current or future media, including reprinting/republishing this material for advertising or promotional purposes, creating new collective works, for resale or redistribution to servers or lists, or reuse of any copyrighted component of this work in other works.

Cite as:

T. Fortin et al., "A Nonlinear Model for Nano-Electro Mechanical Mass Sensing Signals Processing," in IEEE Sensors Journal, vol. 21, no. 19, pp. 21852-21861, 1 Oct.1, 2021, doi:

10.1109/JSEN.2021.3103713.

BibTex:

@ARTICLE $\{9509545$, author $=\{$ Fortin, Thomas and Vysotskyi, Bogdan and Defoort, Martial and Reynaud, Adrien and Lai, Szu-Hsueh and Dominguez-Medina, Sergio and Clement, Kavya and Cumaku, Vaitson and Hentz, Sébastien and Masselon, Christophe\}, journal=\{IEEE Sensors Journal\}, title $=\{$ A Nonlinear Model for Nano-Electro Mechanical Mass Sensing Signals Processing $\}$, year $=\{2021\}$, volume $=\{21\}$, number $=\{19\}$, pages $=\{21852-21861\}$, doi $=\{10.1109 /$ JSEN.2021.3103713 $\}\}$

Abstract link:

https://ieeexplore.ieee.org/document/9509545 


\title{
A nonlinear model for nano-electro mechanical mass sensing signals processing
}

T. Fortin ${ }^{* 1}$, B. Vysotskyi ${ }^{2}$, M. Defoort ${ }^{2}$, A. Reynaud ${ }^{2}$, S.-H. Lai ${ }^{1}$, S. Dominguez-Medina ${ }^{1}$, K. Clement ${ }^{1}$, V. Cumaku ${ }^{1}$, S. Hentz ${ }^{2}$, and C. Masselon ${ }^{1}$

${ }^{1}$ Univ. of Grenoble Alpes, Inserm, CEA, IRIG-BGE, U1092, 38000, Grenoble, France

${ }^{2}$ Univ. Grenoble Alpes, F-38000 Grenoble, France and also with CEA, LETI, MINATEC Campus, F-38054 Grenoble, France

\begin{abstract}
Due to their physical properties, nanomechanical sensors (NEMS) can achieve mass measurements in the mega- to gigadalton range, which is hardly obtained with conventional mass-spectrometers. However, NEMS signals are subject to noise, causing a loss of mass resolution and thus emphasizing the need of noise control. We propose a denoising model that relies on a total variation formulation, which deals with different noise models (particularly colored noise) affecting NEMS. The model also takes into account the physics of NEMS, such as the non-linear coupling between signals of individual NEMS. The performance of the proposed model is tested on simulated data which parameters are chosen similar to true experimental conditions. The obtained results confirm the interest of our model with a mass-resolution increase over $20 \%$ compared to methods used in literature.
\end{abstract}

Keywords: Automatic parameters selection, Colored noise, Mass measurement, Mass-resolution increase, Nanomechanical sensor (NEMS), Nonlinear coupling, Resonance frequency denoising, Total Variation algorithm

\section{Introduction}

A nanomechanical resonator (NEMS) can measure the mass of individual particles accreting on its surface [1]: Since the device's mass is the main parameter which defines resonance frequencies of NEMS oscillations at fixed NEMS stiffness, a particle landing on its surface adds to its total mass and causes downshift

\footnotetext{
*Corresponding author: thomas.fortin@cea.fr
} 
of its resonance frequencies. For the geometry we consider in this report (i.e. doubly-clamped beam), these frequency shifts depend on the particle landing position on the NEMS surface and the frequencies of two resonance modes have to be monitored simultaneously to resolve the two unknowns (i.e., particle mass and position [15]). With successive landing events over time, the frequency traces of the first two resonance modes are thus comparable to piecewise constant time signals where each pair of resonance frequencies downshifts are used to determine the mass and position of a single landed particle.

Authors in 15] proposed a physical model to link simultaneous resonance frequencies downshifts with particles mass and landing position. This model is a system where two unknowns (particle mass $m$ and $i^{\text {th }}$ particle landing position $x_{i}$ ) are linked to the $i^{\text {th }}$ first-resonance-frequency-trace discontinuity $\left[\mathbf{s}^{1}\right]_{i}$, and the $i^{\text {th }}$ second-resonance-frequency-trace discontinuity $\left[\mathbf{s}^{2}\right]_{i}$ through:

$$
\begin{aligned}
& {\left[\mathbf{s}^{1}\right]_{i}=m \phi_{1}\left(x_{i}\right)} \\
& {\left[\mathbf{s}^{2}\right]_{i}=m \phi_{2}\left(x_{i}\right)}
\end{aligned}
$$

where $\phi_{i}$ with $i=1,2$ are known functions.

As a consequence, solving the system of equations (1)-(2) enables to compute particles masses from discontinuities of both NEMS resonance frequency traces. However, solving this system also supposes a perfect knowledge of the time locations and the amplitudes of these discontinuities. While this is not an issue in ideal cases, it becomes less trivial in real conditions: As any actuated devices, NEMS are subject to noise that create artifacts in discontinuities detection but also affect estimates of discontinuities amplitudes. Clearly, artifacts in discontinuities detection as well as poor estimates of discontinuities amplitudes have negative impacts on mass accuracy and resolution when solving system (1)-(2). These impacts should thus be limited as much as possible.

Concerning the problem of artifacts in discontinuities detection, authors in 15 proposed a simple but efficient method: the "ellipse" denoising method. This method is divided into three successive steps.

The first step consists in recording the first NEMS resonance frequency $\mathbf{s}^{1}$ and the second NEMS resonance frequency $\mathbf{s}^{2}$ when no particle deposition occurs, to directly probe the noise affecting the NEMS resonance frequency traces during experiments.

The second step is to draw a scatter plot of $\nabla \mathbf{s}^{2}$ with respect to $\nabla \mathbf{s}^{1}$, where $\nabla \mathbf{s}^{i}$ for $i=1,2$ is defined as all differences of $\mathbf{s}^{i}$ consecutive values. Once the scatter plot has been drawn, it is fitted with a bi-variate Gaussian distribution, thus defining an ellipse encapsulating points only due to noise.

The last step is the true denoising part: While recording NEMS signals when the particle deposition experiment is conducted, both $\nabla \mathbf{s}^{1}$ and $\nabla \mathbf{s}^{2}$ are computed and drawn on the previous scatter plot. If one of the newly acquired 
points lies inside the previously defined ellipse (up to a user's defined threshold), it is not attributed to a particle deposition but to noise effects. Conversely, if the newly acquired point lies outside the ellipse, it is attributed to a particle deposition and thus flagged as such.

As this "ellipse" method leads to satisfactory event detection, we choose not to explore deeper this path and we consider this method as our reference method in discontinuities detection throughout this report. Note that this detection method was used in [1] for data analysis.

By contrast, the problem of poor estimates of discontinuities amplitudes due to noise has not been entirely solved yet: $\sqrt{2}$ proposed a method to remove noise affecting NEMS resonance frequencies but to this aim, introduced numerous additional parameters which can hardly be physically explained; 11 dealt with this discontinuities amplitudes issue by modeling noises as serially uncorrelated random variables ... whereas noises affecting NEMS can also be correlated in time as shown in [2]. Therefore, we decide to focus on this problem and propose here a new denoising model relying on classical Total Variation (TV) algorithms [10 but modified to take into account NEMS specificity. In particular, this model is designed to correct main defects of previously used methods:

1. deal with different type of frequency noise even when correlation in time is not negligible,

2. define parameters directly linked to physical noises properties

3. automatically compute these parameters,

4. include physical features of NEMS, especially the nonlinear coupling between NEMS resonance frequencies

\section{Outline}

After introducing mathematical notations needed to precisely define our model, we will tackle our denoising model. As it largely relies on classical TV models, we decided to split this discussion into three parts, each one being a step towards the final model. We will first describe how to include all noise features for a single NEMS resonance frequency trace. Then, we will describe an intermediate model for two independent NEMS resonance frequency traces but where a new set of variables closely adapted to NEMS physical features is used. At last, we will conclude with the final model focusing on two dependent NEMS resonance frequency traces linked through a nonlinear coupling.

Finally, we will present test cases to measure the efficiency of our new model in comparison with the reference method presented in the introduction. 


\section{Notations}

\subsection{Algebraic notations}

We assume that all matrices and vectors are real. Bold capital letters denote matrices and bold lower-case letters denote column vectors. Lower-case Greek letters always denote scalars.

Space of real matrices with $n$ rows and $m$ columns is denoted $\mathbb{R}^{n \times m}$ and space of column vectors with $n$ rows is denoted $\mathbb{R}^{n}$. The superscript $T$ used with a matrix or a vector denotes the transposed matrix or vector. When minus operator precedes $T$ symbol, it refers to the inverse of the corresponding transposed matrix. Following this notation, the superscript -1 used with a matrix denotes the inverse matrix, if it exists. The coefficient at line $i$ and column $j$ of any matrix $\mathbf{A}$ is denoted $\mathbf{A}_{i j}$. Similar notations are used when dealing with vectors. Euclidean dot-product (respectively norm) for vectors is denoted $<., .>_{2}$ (resp. $\left.\|.\|_{2}\right)$. In a similar way, $L^{1}$ norm for vectors is denoted $\|.\|_{1}$.

Some letters or symbols are reserved for specific vectors and matrices. Thus, $\mathbf{1}_{n}$ (respectively $\mathbf{0}_{n}$ ) denotes a vector in $\mathbb{R}^{n}$ whose all components are equal to 1 (resp. 0). The identity matrix of size $n$ is denoted $\mathbf{I} \mathbf{d}_{n}$. F denotes the discrete Fourier transform matrix. Once an integer $n$ has been set, $\nabla$ will denote the gradient operator that maps a vector of $\mathbb{R}^{n}$ onto $\mathbb{R}^{n-1}$ :

$$
(\nabla \mathbf{s})_{i}:=\mathbf{s}_{i+1}-\mathbf{s}_{i} \quad i=1, \ldots, n-1, \quad \mathbf{s} \in \mathbb{R}^{n}
$$

\subsection{Model notations}

Let $N$ be an integer and $] 1, \ldots, N$ [ the set of all integers strictly greater than 1 and strictly lower than $N$. Let $\mathbb{J}_{N}$ denotes a set of $n<N$ non-equal integers all belonging to $] 1, \ldots, N\left[\right.$. We will suppose that $\mathbb{J}_{N}$ is ordered. We then define $\mathbb{S}_{N}$ as the following set:

$$
\mathbb{S}_{N}:=\left\{\mathbf{s} \in \mathbb{R}^{N} \mid \mathbf{s}_{i}=\mathbf{s}_{i+1} \text { if } i \notin \mathbb{J}_{N}, \mathbf{s}_{i} \neq \mathbf{s}_{i+1} \text { if } i \in \mathbb{J}_{N}\right\}
$$

$\mathbb{S}_{N}$ represents the space of signals which are constant piecewise with discontinuities exactly located at $\mathbb{J}_{N}$. Clearly, $\mathbb{S}_{N}$ has dimension $n+1$ and is the image of $\mathbb{R}^{n+1}$ by a simple matrix $\mathbf{E} \in \mathbb{R}^{N \times(n+1)}$. $\mathbf{E}$ is injective and orthogonal.

Let $[\mathbf{s}]_{i}$ denote the $i$ th $(1 \leq i \leq n)$ discontinuity of any $\mathbf{s} \in \mathbb{S}_{N}$. In other words, we have $[\mathbf{s}]_{i}:=(\nabla \mathbf{s})_{j}$ where $j$ is the $i$ th element of $\mathbb{J}_{N}$. We then denote $\mathbb{D}_{N}$ a subset of $\mathbb{S}_{N}$ whose every element is a signal $\mathbf{s}$ verifying $[\mathbf{s}]_{i} \leq 0$ for all $1 \leq i \leq n$.

We denote $\mathbf{g}(\mathbf{s})$ the gradient-like vector associated to any $\mathbf{s} \in \mathbb{R}^{n+1}$. Its 
definition is given by the following equation:

$$
\mathbf{g}(\mathbf{s})_{i}:= \begin{cases}\mathbf{s}_{1} & \text { if } i=1 \\ (\nabla \mathbf{s})_{i} & \text { otherwise }\end{cases}
$$

A vector $\mathbf{s}$ and its gradient-like vector $\mathbf{g}(\mathbf{s})$ are linked through a gradientlike matrix $\mathbf{G}: \mathbf{s}=\mathbf{G} \mathbf{g}(\mathbf{s})$. This matrix is lower triangular and all its non-zero coefficients are equal to $1: \mathbf{G}$ is thus invertible.

We denote also $\mathbb{G}$ the set of vectors in $\mathbb{R}^{n+1}$ whose components are all nonpositive except the first one:

$$
\mathbb{G}:=\left\{\mathbf{s} \in \mathbb{R}^{n+1} \mid \mathbf{s}_{i} \leq 0 \text { if } i>1\right\}
$$

\section{Modeling}

Let us consider two non-noisy NEMS resonance frequency traces $\mathbf{s}^{1}$ and $\mathbf{s}^{2}$ (see Fig. 1). For the sake of simplicity, we suppose that both traces belong to $\mathbb{D}_{N}$ : The more general case where $\mathbb{D}_{N}$ is replaced by $\mathbb{S}_{N}$ can be treated as in the subsequent paragraphs but with slight adaptations only. Note that a given set $\mathbb{D}_{N}$ (or $\mathbb{S}_{N}$ ) implies the prior knowledge of $\mathbb{J}_{N}$, the set of NEMS resonance frequency traces discontinuities: As mentioned in the introduction, this hypothesis is not unrealistic since we have at our disposal a discontinuitiesdetection method (see [2]).

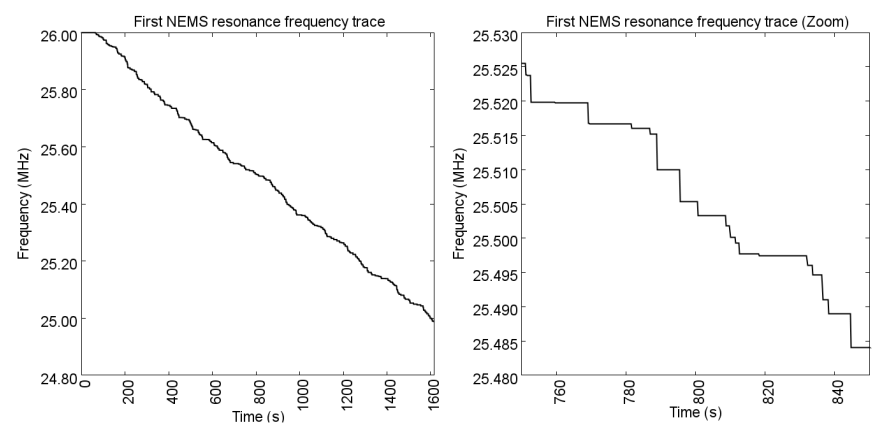

Figure 1: Simulation of the first NEMS resonance frequency trace for a deposition of $106 \mathrm{MDa}$ particles at a average rate of 20 events per minute during 1615s. Total number of particle depositions is equal to 512. Left: trace over 1615s. Right: trace in $[750 \mathrm{~s}, 850 \mathrm{~s}]$ interval (zoom). The trace is piecewise constant over the whole interval.

In fact, $\mathbf{s}^{1}$ and $\mathbf{s}^{2}$ are not recorded exactly and only noisy versions of them, $\mathbf{s}^{\mathbf{n}, 1}$ and $\mathbf{s}^{\mathbf{n}, 2}$, are accessible. For $i=1,2$, each $\mathbf{s}^{\mathbf{n}, i}$ is modeled as the sum of $\mathbf{s}^{i}$ 
with a Gaussian noise $\mathbf{n}^{i}$ of mean 0 and of standard deviation $\sigma^{i}$ :

$$
\begin{aligned}
& \mathbf{s}^{\mathbf{n}, 1}:=\mathbf{s}^{1}+\mathbf{n}^{1} \\
& \mathbf{s}^{\mathbf{n}, 2}:=\mathbf{s}^{2}+\mathbf{n}^{2}
\end{aligned}
$$

\subsection{Model for a single NEMS resonance frequency trace}

To denoise a single noisy NEMS resonance frequency trace $\mathbf{s}^{\mathbf{n}}$, we propose to rely on a TV minimization model. The latter consists in denoising $\mathbf{s}^{\mathbf{n}}$ by finding a trace $\hat{\mathbf{s}}$ solution of the minimization problem (3):

$$
\underset{\substack{\mathbf{v} \in \mathbb{D}_{N} \\\left\|\mathbf{v}-\mathbf{s}^{\mathbf{n}}\right\|_{\mathbf{2}} \leq \sigma}}{\arg \min }\|\nabla \mathbf{v}\|_{1}
$$

This model is chosen because it has numerous advantages when considering the physical features of a NEMS resonance frequency trace. First, solving (3) provides a signal estimate $\hat{\mathbf{s}}$ which is naturally piecewise constant, in agreement with one of the main feature of a NEMS resonance frequency trace. Second, (3) ensures $\hat{\mathbf{s}}$ is the piecewise constant signal with the smallest discontinuities that can explain $\mathbf{s}^{\mathbf{n}}$. Since the particle mass is directly connected to NEMS frequency trace discontinuities through (1)-(2), it seemed natural to use a method in which signal discontinuities are central. At last, $(3)$ has been largely studied in theory as well as in practice $([5],[6],[10])$.

However the quadratic constraint in (3) is dedicated to the sole case of white Gaussian noise, and thus (3) cannot be used when the noise is not entirely defined by its standard deviation, in other words when the noise is time-correlated or colored. As the noise affecting NEMS can also be colored ( [2], [12]), we have to adapt (3) to fit with a more general case.

A colored noise $\mathbf{n}$ can always be modeled such that every component $\mathbf{n}_{i}$ for $i=1, \ldots, N$ is the convolution in the time domain of a given white noise $\mathbf{n}^{w}$ and a fixed convolution kernel $\mathbf{h}$ : This relationship exactly writes $\mathbf{n}_{i}:=$ $\sum_{k=1}^{N} \mathbf{h}_{k-i} \mathbf{n}_{k}^{w}$ for $i=1, \ldots, N$. In the Fourier domain, the previous equation equivalently rewrites $(\mathbf{F n})_{i}=(\mathbf{F h})_{i}\left(\mathbf{F} \mathbf{n}^{w}\right)_{i}$ for $i=1, \ldots, N$. Since the Power Spectral Density (PSD) of a white noise is by definition equal to a constant, we deduce that the PSD of $\mathbf{n}$ is proportional to the PSD of $\mathbf{h}$. Conversely, applying the same arguments in reverse order allows to compute the PSD of a noise $\mathbf{n}^{w}$ from the PSD of any given colored noise $\mathbf{n}$, once the PSD of the convolution kernel $\mathbf{h}$ is known.

This remark leads us to the following idea: We propose to define a matrix $\mathbf{W}$ which transforms a given colored Gaussian noise $\mathbf{n}$ into a white Gaussian noise $\mathbf{W n}$, and to apply the constraint in (3) not to $\mathbf{n}$ but rather to $\mathbf{W n}$. With such a transform, the colored noise affecting NEMS would be taken into account through the structure and coefficient values of $\mathbf{W}$. 
Consequently, let's consider a diagonal matrix $\mathbf{D}$ which coefficients will be defined afterward and the new Gaussian noise Wn with $\mathbf{W}:=\mathbf{D F}$. Moreover, let us assume there are two constants $\alpha$ and $\beta$ independent of $N$ such that $\alpha\|\mathbf{v}\|_{\mathbf{2}} \leq\|\mathbf{D} \mathbf{v}\|_{\mathbf{2}} \leq \beta\|\mathbf{v}\|_{\mathbf{2}}$ for every $\mathbf{v}$ belonging to $\mathbb{R}^{N}$.

Since a white Gaussian noise has uniform PSD, a solution would be to compute $\mathbf{D}$ such that $\left|(\mathbf{W n})_{i}\right|^{2}$ is also constant for every $i=1, \ldots, N$. D being diagonal, it is also equivalent to find the coefficients of matrix $\mathbf{D}$ such that $\left|\mathbf{D}_{i i}\right|^{2}\left|(\mathbf{F n})_{i}\right|^{2}$ is constant. But $\left|(\mathbf{F n})_{i}\right|^{2}$ is the PSD of colored noise $\mathbf{n}$ : This PSD can be estimated either based on a model or using prior noise measurements. As a consequence, $\mathbf{D}$ coefficients are easy to compute for the equivalent white noise $\mathbf{W n}$.

We are now able to modify (3) to deal with colored Gaussian noise. To this end, we propose the following model:

$$
\underset{\substack{\mathbf{v} \in \mathbb{D}_{N} \\\left\|\mathbf{W}\left(\mathbf{v}-\mathbf{s}^{\mathbf{n}}\right)\right\|_{\mathbf{2}} \leq \sigma}}{\arg \min }\|\nabla \mathbf{v}\|_{1}
$$

where $\sigma$ is the standard-deviation of the equivalent white noise $\mathbf{W n} . \sigma^{c}$ is the standard-deviation of the colored noise where:

$$
\sigma^{c}=\sigma \sqrt{\frac{1}{N} \sum_{i=1}^{N} \frac{1}{\mathbf{D}_{i i}^{2}}}
$$

Remark 1 Interestingly, problem (4) depends on matrix $\boldsymbol{D}$ up to a multiplicative constant. This property has practical consequences: For colored noise whose transfer functions have simple shapes (such as pink noise), determining coefficients of matrix $\boldsymbol{D}$ from experiments is reduced to only determining a small number of parameters up to a multiplicative constant.

Constrained problems such as (4) are rarely solved in this form and most people prefer to tackle equivalent augmented-Lagrangian formulations which are easier to solve numerically. With this kind of method, the quadratic constraint in constrained models is removed and replaced by an additional quadratic term into the function to minimize. For instance, minimization problem (4) formally becomes minimization problem (6), where $\lambda$ is a parameter to set:

$$
\underset{\mathbf{v} \in \mathbb{D}_{N}}{\arg \min }\|\nabla \mathbf{v}\|_{1}+\frac{\lambda}{2}\left\|\mathbf{W}\left(\mathbf{v}-\mathbf{s}^{\mathbf{n}}\right)\right\|_{2}^{2}
$$

The main issue then relies on proving a mathematical equivalence between the original constrained model and the final augmented-Lagrangian model i.e. in our case, between (4) and (6). For (3), this equivalence has been proved in [6] which suggests that it is also possible for (4). With slight adaptations, tools developed in 6 can indeed be used and provide an equivalence between (6) and (4) if $\lambda$ is in a one-to-one mapping with the noise standard deviation 
$\sigma$. Nevertheless, the function which links the correct value of $\lambda$ with the value of $\sigma$ cannot be known analytically. Thus, ensuring exact equivalence between (4) and (6) seems to be a dead-end. This issue has fortunately been solved in [5] (for $\mathbf{W}=\mathbf{I d}_{N}$ ) and in [4] (for a general matrix $\mathbf{W}$ ): If $\lambda$ cannot be directly computed, it can yet be computed as the limit of some simple iterative procedures. In short, $\lambda$ depends only on the noise parameter $\sigma$ and can be computed automatically, up to a chosen accuracy.

Remark 2 Note that the minimization problem (6) can also be seen as an a posteriori validation test for the discontinuities detection method. Indeed, let us denote $\hat{\boldsymbol{v}}$ the solution of (6) and $\overline{\boldsymbol{v}}$ the solution of the minimization problem (7):

$$
\underset{\boldsymbol{v} \in \mathbb{D}_{N}}{\arg \min }\left\|\boldsymbol{W}\left(\boldsymbol{v}-\boldsymbol{s}^{\boldsymbol{n}}\right)\right\|_{2}^{2}
$$

Minimization problem (7) is easy and fast to solve since it is purely quadratic. Moreover, since $\left\|\boldsymbol{W}\left(\overline{\boldsymbol{v}}-\boldsymbol{s}^{\boldsymbol{n}}\right)\right\|_{2}$ is lower than $\left\|\boldsymbol{W}\left(\hat{\boldsymbol{v}}-\boldsymbol{s}^{\boldsymbol{n}}\right)\right\|_{2}$ by definition of $\overline{\boldsymbol{v}}$, we should also have $\left\|\boldsymbol{W}\left(\overline{\boldsymbol{v}}-\boldsymbol{s}^{\boldsymbol{n}}\right)\right\|_{2} \leq \sigma$. If this inequality is not verified, it clearly means that the space $\mathbb{D}_{N}$ is too small or in other words, that the number of discontinuities given by the discontinuities detection method is too small: A less restrictive discontinuities detection threshold should therefore be used.

\subsection{Model for two uncoupled NEMS resonance frequency traces}

The easiest way to generalize a TV minimization model to simultaneously smooth two uncoupled NEMS resonance frequency traces is to compute two estimates $\hat{\mathbf{s}}^{1}$ and $\hat{\mathbf{s}}^{2}$ of true signals $\mathbf{s}^{1}$ and $\mathbf{s}^{2}$ as solutions of:

$$
\underset{\left(\mathbf{v}^{1}, \mathbf{v}^{2}\right) \in \mathbb{D}_{N}^{2}}{\arg \min }\left\|\nabla \mathbf{v}^{1}\right\|_{1}+\left\|\nabla \mathbf{v}^{2}\right\|_{1}+\frac{\lambda_{1}}{2}\left\|\mathbf{W}^{1}\left(\mathbf{v}^{1}-\mathbf{s}^{\mathbf{n}, 1}\right)\right\|_{2}^{2}+\frac{\lambda_{2}}{2}\left\|\mathbf{W}^{2}\left(\mathbf{v}^{2}-\mathbf{s}^{\mathbf{n}, 2}\right)\right\|_{2}^{2}
$$

Indeed, model (8) straightforwardly splits into two independent TV minimization models (6), if signals $\hat{\mathbf{s}}^{1}$ and $\hat{\mathbf{s}}^{2}$ can be considered independent.

Since every $\mathbf{v}^{1}$ and every $\mathbf{v}^{2}$ in $(8)$ belong to $\mathbb{D}_{N}$, both direction and time location of signals discontinuities are known. As a consequence, absolute values in (8) can be dropped and $L^{1}$ terms are in fact linear terms. Conversely, $L^{2}$ terms can be rewritten as dot-products so that, gathering all these terms with their new formulation, we are able to rewrite $(8)$ as a simple constrained quadratic (CQ) model:

$$
\underset{\left(\mathbf{v}^{1}, \mathbf{v}^{2}\right) \in \mathbb{D}_{N}^{2}}{\arg \min } \frac{1}{2}\left\langle\left(\begin{array}{cc}
\mathbf{A}^{1} & \mathbf{0} \\
\mathbf{0} & \mathbf{A}^{2}
\end{array}\right)\left(\begin{array}{c}
\mathbf{v}^{1} \\
\mathbf{v}^{2}
\end{array}\right),\left(\begin{array}{c}
\mathbf{v}^{1} \\
\mathbf{v}^{2}
\end{array}\right)\right\rangle_{2}-\left\langle\left(\begin{array}{l}
\mathbf{b}^{1} \\
\mathbf{b}^{2}
\end{array}\right),\left(\begin{array}{c}
\mathbf{v}^{1} \\
\mathbf{v}^{2}
\end{array}\right)\right\rangle_{2}
$$

where we have set for $k=1,2$ :

$$
\mathbf{A}^{k}:=\lambda_{k} \mathbf{W}^{k^{\top}} \mathbf{W}^{k}
$$




$$
\mathbf{b}^{k}:=\left(\begin{array}{c}
-1 \\
\mathbf{0}_{N-2} \\
1
\end{array}\right)+\mathbf{A}^{k} \mathbf{s}^{\mathbf{n}, k}
$$

We then replace vectors $\mathbf{v}^{j}$ for $j=1,2$ in equation $(9)$ by their discontinuities $\left[\mathbf{v}^{j}\right]_{i}$ for $i=1, \ldots, n$ : This step is necessary to allow further developments on NEMS resonance frequencies coupling. To this aim, we first notice that for every $\mathbf{v} \in \mathbb{R}^{N}$, there is a unique vector $\mathbf{r} \in \mathbb{R}^{n+1}$ such that:

$$
\mathbf{v}:=\mathbf{E r}
$$

This mapping offers two important advantages: It drastically reduces the number of degrees of freedom (from $N$ to $n+1$ ) and discontinuities of $\mathbf{v}$ verify $[\mathbf{v}]_{i}=(\nabla \mathbf{r})_{i}$ for $i=1, \ldots, n$. The next step is to rewrite $\mathbf{r}$ in term of its gradient to get the desired change of variable. Using our notations, the vector $\mathbf{r}$ is rewritten as:

$$
\mathbf{r}=\mathbf{G g}(\mathbf{r})
$$

Gathering both relationships, we finally find that $\mathbf{v}=\mathbf{E G g}(\mathbf{r})$. Note that $\mathbf{g}(\mathbf{r})$ has a special structure due to the fact that $\mathbf{v}$ belongs to $\mathbb{D}_{N}$ : All its components but the first one are non-positive and thus $\mathbf{g}(\mathbf{r}) \in \mathbb{G}$. Applying this last algebraic relation to vectors $\mathbf{v}^{j}$ for $j=1,2$ in $(9)$, we are finally led to the following minimization problem whose solutions $\widehat{\mathbf{g}(\mathbf{r})}^{1} \in \mathbb{G}$ and $\widehat{\mathbf{g}(\mathbf{r})}^{2} \in \mathbb{G}$ are estimates of NEMS resonance frequencies discontinuities amplitudes:

$$
\underset{\left(\mathbf{q}^{1}, \mathbf{q}^{2}\right) \in \mathbb{G}^{2}}{\arg \min } \frac{1}{2}\left\langle\left(\begin{array}{cc}
\widetilde{\mathbf{A}}^{1} & \mathbf{0} \\
\mathbf{0} & \widetilde{\mathbf{A}}^{2}
\end{array}\right)\left(\begin{array}{c}
\mathbf{q}^{1} \\
\mathbf{q}^{2}
\end{array}\right),\left(\begin{array}{c}
\mathbf{q}^{1} \\
\mathbf{q}^{2}
\end{array}\right)\right\rangle_{2}-\left\langle\left(\begin{array}{c}
\widetilde{\mathbf{b}}^{1} \\
\widetilde{\mathbf{b}}^{2}
\end{array}\right),\left(\begin{array}{c}
\mathbf{q}^{1} \\
\mathbf{q}^{2}
\end{array}\right)\right\rangle_{2}
$$

where we have set for $k=1,2$ :

$$
\begin{gathered}
\widetilde{\mathbf{A}}^{k}:=\mathbf{G}^{\top} \mathbf{E}^{\top} \mathbf{A}^{k} \mathbf{E} \mathbf{G} \\
\widetilde{\mathbf{b}}^{k}:=\left(\begin{array}{c}
0 \\
\mathbf{1}_{n}
\end{array}\right)+\mathbf{G}^{\top} \mathbf{E}^{\top} \mathbf{A}^{k} \mathbf{s}^{\mathbf{n}, k}
\end{gathered}
$$

Model 10 is a CQ model the constraints of which are very simple: Indeed, they only impose, for vectors in $\mathbb{R}^{n+1}$, non-positive values on all components but the first one. This kind of constrained problem can be solved with iterative algorithms: Projected steepest gradient algorithm is perfectly adapted or to get estimates even faster, the more recent Nesterov's algorithm can be also used [8], [9]. Note that for both these iterative algorithms, every iterate exactly verifies the non-positive constraints. When user's accuracy threshold is reached, every signal discontinuity is consistently estimated, which should lead (after applying resonance-frequencies-discontinuities-to-mass mapping) to a smaller number of inconsistent mass estimates. This is another argument in favor of the proposed change of variables. 


\subsection{Model for two coupled NEMS resonance frequency traces}

Let us now take into account the coupling between NEMS resonance frequency traces $\mathbf{s}^{1}$ and $\mathbf{s}^{2}$. According to the system of equations (1)-(2), $\mathbf{s}^{1}$ is linked to $\mathbf{s}^{2}$ through a known real $\psi_{0}$ and a known function $\Psi$ which only depends on particle landing positions:

$$
\left\{\begin{array}{l}
\mathbf{s}_{1}^{2}:=\psi_{0} \mathbf{s}_{1}^{1} \\
{\left[\mathbf{s}^{2}\right]_{i}=\Psi\left(x_{i}\right)\left[\mathbf{s}^{1}\right]_{i} \quad i=1, \ldots, n}
\end{array}\right.
$$

where $x_{i}$ denotes the particle landing position corresponding to the $i$ th discontinuity in $\mathbb{J}_{N}$.

This equation suggests to introduce $x_{i}$ as a variable in our desired nonlinear model to deal with resonance frequencies coupling. But in doing so, we introduce an additional degree of complexity: Indeed, the use of the function $\Psi$ requires additional computations at every step of our iterative process. However it is not strictly necessary to get a perfect signal estimate. It has been shown in [2] that despite its complex shape, function $\Psi$ is a one-to-one mapping between possible landing positions interval $\left[0, \frac{1}{2}\right]$ and a known interval $\mathbb{I}$, the form of which is $\mathbb{I}:=\left[0, \psi_{\max }\right]$. As a consequence, it is possible to replace every $\Psi\left(x_{i}\right)$ with a number $\psi_{i}$ belonging to $\mathbb{I}$ : The cost of our algorithm is thus less important and if landing positions are needed in further applications, they can be computed after signal denoising has been performed through solving the inverse problem $\psi_{i}=\Psi\left(x_{i}\right)$. Moreover, since $\mathbb{I}$ is a finite and closed interval, the corresponding constraint on $\psi_{i}$ is very easy to deal with in our TV minimization model.

Let us modify (10) to include resonance frequencies coupling. Recalling that $\mathbf{s}^{k}:=\mathbf{E G} \mathbf{g}\left(\mathbf{r}^{k}\right)$ for $k=1,2$, we define a diagonal matrix $\boldsymbol{\Psi}$ such that:

$$
\mathbf{g}\left(\mathbf{r}^{2}\right)=\mathbf{\Psi g}\left(\mathbf{r}^{1}\right)
$$

Using signals coupling equations, $\boldsymbol{\Psi}$ coefficients verify the following equations:

$$
\left\{\begin{array}{l}
\boldsymbol{\Psi}_{11}:=\psi_{0} \\
\boldsymbol{\Psi}_{i i}:=\psi_{i-1} \quad i=2, \ldots, n+1
\end{array}\right.
$$

As a consequence, equation (12) suggests to use $\mathbf{g}\left(\mathbf{r}^{1}\right)$ and $\boldsymbol{\Psi}$ as variables in (10) instead of the gradient-like variables $\mathbf{g}\left(\mathbf{r}^{1}\right)$ and $\mathbf{g}\left(\mathbf{r}^{2}\right)$. In doing so, model (10) becomes the nonlinear model 13 , whose solutions are simply denoted $\widehat{\mathbf{g}(\mathbf{r})} 1$ and $\widehat{\Psi}$ :

$$
\underset{\left(\mathbf{q}^{1}, \mathbf{\Phi}\right) \in \mathbb{G} \times \mathbb{P}}{\arg \min } \frac{1}{2}\left\langle\left(\widetilde{\mathbf{A}}^{1}+\boldsymbol{\Phi}^{\top} \widetilde{\mathbf{A}}^{2} \mathbf{\Phi}\right) \mathbf{q}^{1}, \mathbf{q}^{1}\right\rangle_{2}-\left\langle\widetilde{\mathbf{b}}^{1}+\boldsymbol{\Phi}^{\top} \widetilde{\mathbf{b}}^{2}, \mathbf{q}^{1}\right\rangle_{2}
$$

In (13), $\mathbb{P}$ stands for the space of diagonal matrices the non-zero coefficients of which all belong to $\mathbb{I}$ but the first one; matrices and vectors attached to the 
first signal (i.e. with superscript 1) and the second signal (i.e. with superscript 2 ) are unmodified in comparison with (10).

This change of variables has important effects on both solution estimates and numerical methods to compute them. First, the non fully quadratic nature of our coupling model implies that we will only reach a local minimum and not necessarily the optimum: As a consequence, the question arises of how to increase the chance of getting the global minimum among all the local minima. Second, we need to redesign our iterative algorithm since projected steepest gradient method as well as Nesterov's method are designed for pure CQ models.

Let us consider the problem of a global and local minima. To enhance our chances to reach a global optimum, iterative algorithms dedicated to solving (13) have to be initialized with a starting point already close to the desired global minimum. If no true guarantee can be given with such a choice, a starting point sets with solutions of model $(10)$ seems both natural and efficient: Indeed, solutions of (10) already satisfy almost all requirements of (13) but are also unique and easy to compute due to the quadratic nature of 10 . A slight transformation is nevertheless needed to make solutions of $(10)$ acceptable starting points for 13 . The solution $\widehat{\Psi}$ of $(13)$ has to be estimated by solving $\sqrt{12}$, where $\Psi$ is the variable belonging to $\mathbb{P}$ and where $\mathbf{g}\left(\mathbf{r}^{1}\right)$ and $\mathbf{g}\left(\mathbf{r}^{2}\right)$ are equal to the previously computed solutions of $(10)$.

Let us now focus on the non fully quadratic nature of (13). A closer look at it shows that if $\mathbf{q}^{1}$ (respectively $\boldsymbol{\Phi}$ ) is set as a given fixed parameter, the resulting problem is then quadratic in the single variable $\boldsymbol{\Phi}$ (resp. $\mathbf{q}^{1}$ ) and thus, can be solved using either the projected steepest gradient method or Nesterov's method. This property suggests not to solve $(13)$ as as whole but rather to split it into two single-variable quadratic problems (one dedicated to $\mathbf{q}^{1}$, the other to $\boldsymbol{\Phi})$ and solve them consecutively, until reaching simultaneous stationarity in both variables. Using an alternating direction algorithm such as Algorithm 1 is then perfectly suitable.

The nonlinear model proposed here respects the physics of NEMS: We derived a model where the coupling between the first and the second resonance frequencies is explicit and where the intensity of this coupling is also controlled and lies in the right interval. Besides, Algorithm 1 ensures that this coupling and its intensity will be exactly taken into account in every iterative step, thanks to the simplicity of the constraint $\boldsymbol{\Phi} \in \mathbb{P}$. Note also that the way we took into account the nonlinear coupling between NEMS frequency traces is original: To our knowledge, the closest method was described in $[3]$ and relied on a Bayesian formulation, which requires additional parameters compared to the proposed method and is more complex. 


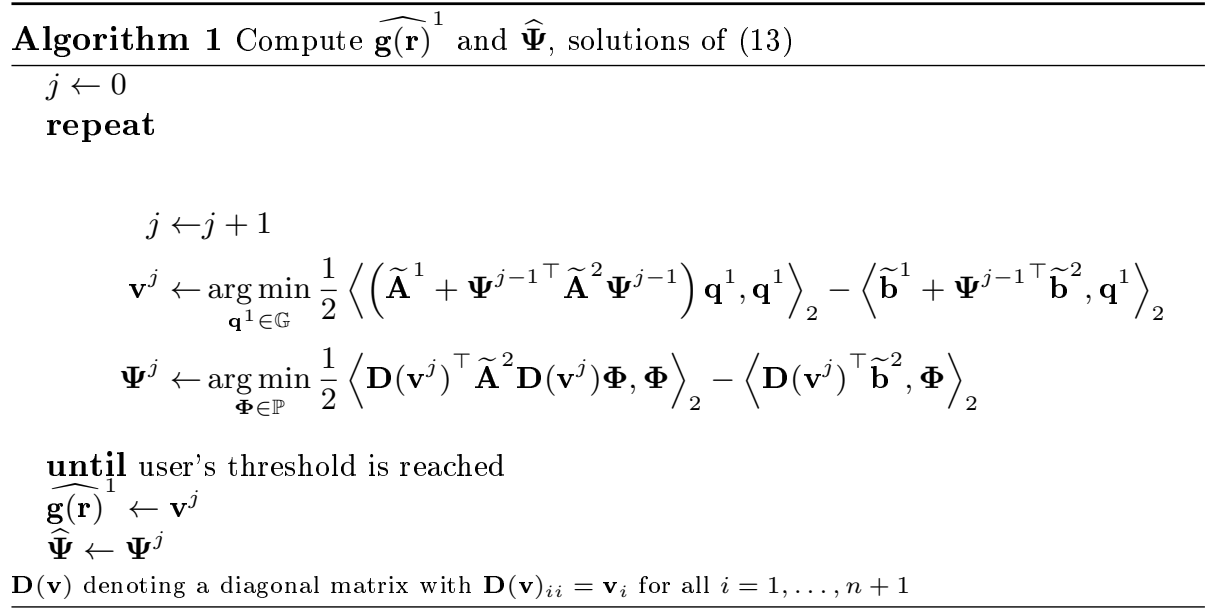

\section{$5 \quad$ Numerical experiments}

In the subsequent paragraphs, we will show a comparison between a reference denoising model used in [1] and the model proposed in this report. Since our goal is to provide a denoising method whose effects imply a better mass estimate and/or mass resolution, all results will be expressed in terms of gain/loss of particles mass estimations and not in terms of signal-denoising efficiency.

\subsection{Tests description}

To test the effect of our denoising model, we generated two simulated NEMS resonance frequency traces $\mathbf{s}^{1}$ and $\mathbf{s}^{2}$ which correspond to depositions of 106 MDa (Mega Dalton) particles at an average rate of 20 events per minute during 1615 seconds (see Fig. 1): The exact number of particle deposited is equal to 512. The first and second NEMS frequencies prior to the particles deposition are respectively equal to $2.610^{7} \mathrm{~Hz}$ and $7.04610^{7} \mathrm{~Hz}$. This set of parameters has been chosen to approach values commonly observed in experimental conditions. Note also that the $106 \mathrm{MDa}$ mass corresponds to the average mass of the bacteriophage $T 5$ which is studied in our laboratory.

Signals $\mathbf{s}^{1}$ and $\mathbf{s}^{2}$ are simulated by using equations (1)-(2) where mass $m$ and landing positions $x_{i}$ are not unknowns but given parameters. If $m$ is set to 106 MDa, the set of landing positions requires a method to be generated: We used a uniform probability distribution on the possible normalized landing positions interval $[0,1]$ to generate every $x_{i}$. Time locations of frequency traces discontinuities are determined with an exponential distribution of rate parameter equal to 20 events per minute. All these parameters being set, the corresponding mass distribution is illustrated in Fig. 2. Note that for the sake of completeness, we also draw a graph on Fig. 2 showing the nonlinear coupling 12 for the same parameters set. 

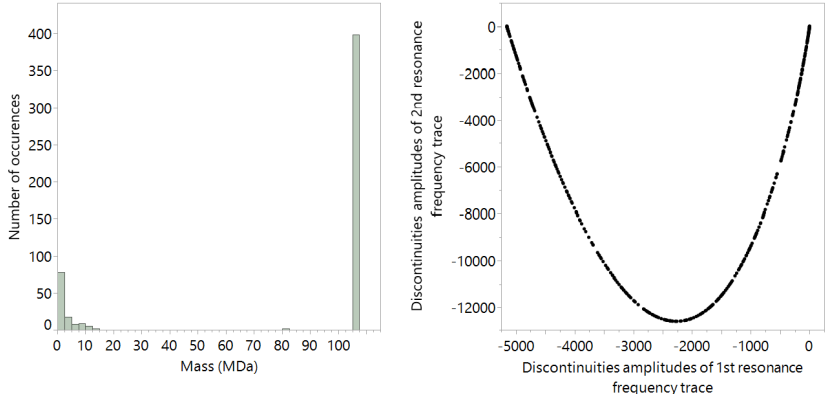

Figure 2: Left: Histogram of mass distribution computed from exact NEMS resonance frequency traces shown in Fig. 1. Low masses are due to the illconditioning of the system (1)-(2) which implies poor mass resolution for some particle landing positions. Right: Graph showing the nonlinear coupling between discontinuities amplitudes of both NEMS frequency traces with no perturbations due to noise.

We then generated two Gaussian noises $\mathbf{n}^{1}$ and $\mathbf{n}^{2}$ and added them to $\mathbf{s}^{1}$ and $\mathbf{s}^{2}$ respectively: Resulting frequencies $\mathbf{s}^{\mathbf{n}, 1}$ and $\mathbf{s}^{\mathbf{n}, 2}$ are the ones to be denoised to get particles mass estimates. Noise features are thus known a priori: They will be described below for every test case.

Estimating the efficiency of the proposed denoising model for particle mass estimation required additional tools: a reference denoising model, a fixed set of detected frequency traces discontinuities $\mathbb{J}_{N}$ to enable unbiased comparisons and a frequencies-discontinuities-to-mass conversion method.

Selecting $\mathbb{J}_{N}$ as well as computing particles masses from $\mathbb{J}_{N}$ are methods extensively described in [2]: As we will use them as predefined tools, we will not go into further details and advise interested readers to refer to this document for technical explanations.

The denoising method we choose as a reference is the one used in [1]: For the $i$ th discontinuity in $\mathbb{J}_{N}$, corresponding discontinuities amplitudes are simply set as $\left[\mathbf{s}^{\mathbf{n}, 1}\right]_{i}$ for the first NEMS resonance frequency trace and $\left[\mathbf{s}^{\mathbf{n}, 2}\right]_{i}$ for the second NEMS resonance frequency trace. It means that the reference method does not apply any filtering on recorded frequencies before mass estimation. Despite its simplicity, this way of estimating discontinuities amplitudes is yet consistent: As the number of detected particles deposited increases, the mean of the particles mass distribution will converge towards the expected value (106 MDa here). However we expect its resolution (i.e. the spread of the computed mass distribution around its mean) to be influenced by noise level and features.

To get masses from resonance frequency traces discontinuities, the nonlinear system of equations (1)-(2) has to be solved: Since $\phi_{1}$ and $\phi_{2}$ functions are com- 
plex, it can only be done using numerical algorithms. Therefore, we decided to use a classical L-BFGS-B algorithm ( $[13,[14]$ ) because of its low memory requirements and its ability to handle simple constraints, but every quasi-Newton algorithm which deals with simple constraints would work as well.

Once NEMS data have been converted into masses, we applied an additional filter to the computed masses. This filtering is a consequence of the mathematical properties of the system (1)-(2), which derives from the NEMS geometry and physical properties. For a doubly-clamped-beam NEMS for instance, the portions of the beam close to a clamp have low sensitivity due to the nearby constraint [2], [15]. When a particle landing position is close to a clamp, the NEMS low sensitivity is translated into an ill-conditioning of the system (1)-(2) at the corresponding position: A small error in the computation of this position is amplified into a huge error in mass estimate.

As we use numerical algorithms with finite precision by essence, errors in positions computations are unavoidable and very high/very low non-physical masses are then obtained. Note that this error amplification effect is solely due to the NEMS itself and thus, even occurs in the absence of noise affecting the NEMS traces, or with any other choice of nonlinear solving algorithm (see Fig. 2).

Consequently, some computed positions have to be discarded to keep a good mass resolution: Authors in $[2$ and $[15]$ showed that the best resolution in term of mass is obtained for particles that land on the position intervals $[0.27,0.48]$ and $[0.52,0.73]$ (for a total beam length normalized to 1 ). Following these reports, we chose to discard particles whose computed landing positions lie outside $[0.27,0.48] \cup[0.52,0.73]$ and to keep for analysis only particles whose computed landing positions are in $[0.27,0.48] \cup[0.52,0.73]$. The position filter effect is shown in Fig. 3 where the mass histogram computed from signals $\mathbf{s}^{1}$ and $\mathbf{s}^{2}$ does not have inconsistent mass estimates anymore: 

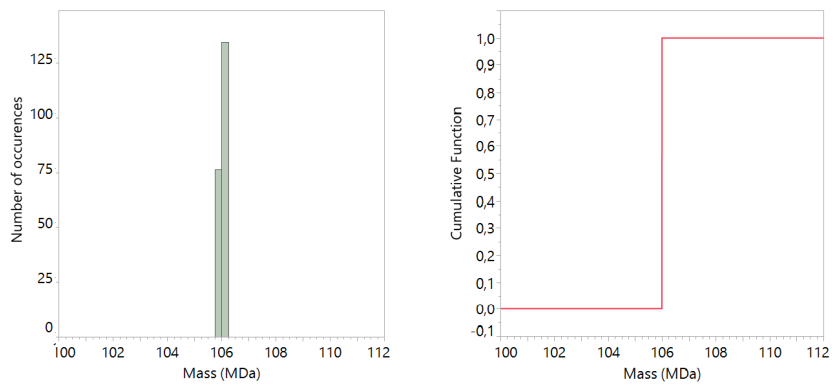

Figure 3: Histogram (4 bins per mass unit) and cumulative function of mass distribution computed from exact NEMS resonance frequency traces shown in Fig. 1. after applying a filter on particle landing positions: particles landing outside $[0.27,0.48] \cup[0.52,0.73]$ are not taken into account because of a low mass accuracy. The mean of the resulting mass distribution $\mu$ is equal to $106 \mathrm{MDa}$ and the standard deviation $\sigma$ is equal to $1.210^{-3} \mathrm{MDa}$. Note that the number of mass events in the range $[100,112] \mathrm{MDa}$ is lower (210) than the expected value (512).

\subsection{Tests validation}

\subsubsection{White noise case}

For this test case, noise $\mathbf{n}^{1}$ and $\mathbf{n}^{2}$ are white Gaussian noise with zero mean and standard deviation $\sigma_{1}=45.76 \mathrm{~Hz}$ and $\sigma_{2}=125.38 \mathrm{~Hz}$ respectively. These values are representative of measured means and standard deviations in experiments. To measure how noise impact NEMS resonance frequency traces discontinuities, we defined an equivalent to the classical Signal-to-Noise-Ratio (which will also be denoted SNR in the following): For a given resonance frequency, this indicator is computed as the ratio of trace discontinuities power when the NEMS trace is not affected by noise to trace discontinuities power when the NEMS trace is affected by noise. In the present case, SNR is equal to 1.001 for the first NEMS resonance frequency and is equal to 0.996 for the NEMS second resonance frequency.

Figure 4 shows the obtained mass distribution for our model as well as for the reference model after all data processing steps. 

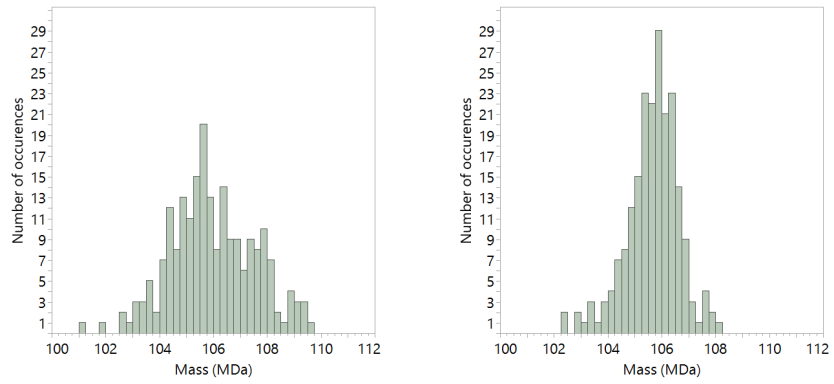

Figure 4: Case of white Gaussian noise with means equal to $0 \mathrm{~Hz}$ and standard deviations equal to $45.76 \mathrm{~Hz}$ and $125.39 \mathrm{~Hz}$. Left: Histogram of mass distribution computed from reference model ( 4 bins per mass unit). The mean of the resulting mass distribution is equal to $105.95 \mathrm{MDa}$ and its standard deviation is equal to $1.56 \mathrm{MDa}$. The number of mass events in the range [100,112] MDa is equal to 211. Right: Histogram of mass distribution computed from proposed model (4 bins per mass unit). The mean of the resulting mass distribution is equal to $105.66 \mathrm{MDa}$ and its standard deviation is equal to 0.97 MDa. The number of mass events in the range $[100,112] \mathrm{MDa}$ is equal to 210.

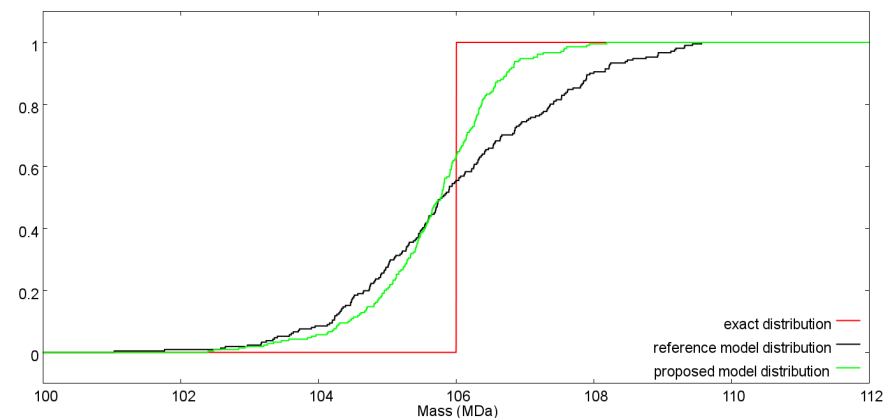

Figure 5: Case of white Gaussian noise with means equal to $0 \mathrm{~Hz}$ and standard deviations equal to $45.76 \mathrm{~Hz}$ and $125.39 \mathrm{~Hz}$. Cumulative functions of mass distribution computed from exact distribution, reference method and proposed method.

Table 1 summarizes main results of computed mass distributions for the different methods : 


\begin{tabular}{|c|c|c|c|}
\hline & expected values & reference model & proposed model \\
\hline mean (MDa) & 106 & 105.95 & 105.66 \\
\hline std dev. (MDa) & $1.210^{-3}$ & 1.56 & 0.97 \\
\hline nb events & 210 & 211 & 210 \\
\hline
\end{tabular}

Table 1: Table of mass distribution statistics for exact case, reference method case and proposed method case for the white Gaussian noise described in Fig. 4.

At this stage, some comments have to be made on table 1. Considering the mean of computed mass distributions, we see that the reference method provides better results. But the loss of accuracy for our model is still acceptable: The relative error on the mass distribution mean for our method is indeed $0.32 \%$ compared to $0.05 \%$ for the reference method. In contrast, all other criteria are in favor of our method as expected: We get a $37,8 \%$ gain in term of standard deviation.

We then explain why we will focus our interpretation on empirical cumulative distributions in the following. Empirical cumulative distributions are efficient concepts in the present situation since they avoid two interpretation biases and have some useful mathematical properties.

First, their plotting is independent on any user's parameter choice unlike histograms with the bin size issue. As a consequence, a data analysis that relies on empirical cumulative distributions is more robust and free from visual bias. In the present report, we kept histograms for visual purposes only.

Second, empirical cumulative distributions allow comparisons between different probability distributions without requiring prior knowledge of these distributions. Without cumulative functions, we could have been tempted to choose arbitrarily a probability distribution which fits our data and then try to analyze them with respect to this choice. As cumulative distributions do not require to know the underlying distributions of the data, we are not subjected to interpretation biases.

Let us focus on Fig. 5 where the exact, reference model and proposed model cumulative distributions are plotted. We first observe that the distance between the cumulative distribution of the proposed model and the exact cumulative distribution is less than the distance between the cumulative distribution of the reference model and the exact cumulative distribution: Our model thus better approximates the data underlying probability distribution than the reference model. Moreover, the cumulative distribution of the proposed model is sharper than in the reference model. It confirms that the standard deviation is smaller with our model and reinforces the standard deviation comparison in table 1 as well as our visual impression from Fig. 4 .

\subsubsection{Pink noise case}

As pink noise depends not only on its mean and standard deviation but also on its PSD, we show here two examples where the differences between reference method and proposed method are emphasized. Each pink noise has been gen- 
erated from a known white noise convoluted by a given transfer function, which enforces the resulting PSD to behave as expected. For the sake of completeness, we will provide this transfer function, original-white-noise main features and the final PSD every time.

Let us first consider pink noise for first and second NEMS resonance frequencies whose transfer functions and PSD are shown in fig 6 . Their means are both equal to $0 \mathrm{~Hz}$ and their standard deviations are equal to $45.36 \mathrm{~Hz}$ and 125.13 $\mathrm{Hz}$ respectively, in agreement with the observed values in experiments. The corresponding SNR is equal to 1.001 for the first NEMS resonance frequency and is equal to 0.996 for the NEMS second resonance frequency. These noises have pretty much the same energies as the white Gaussian noises above, so results can thus be compared without bias. Note also that the pink noise shown here were generated from white noise of mean $0 \mathrm{~Hz}$ and standard deviations 28.94 $\mathrm{Hz}$ and $73.5 \mathrm{~Hz}$.
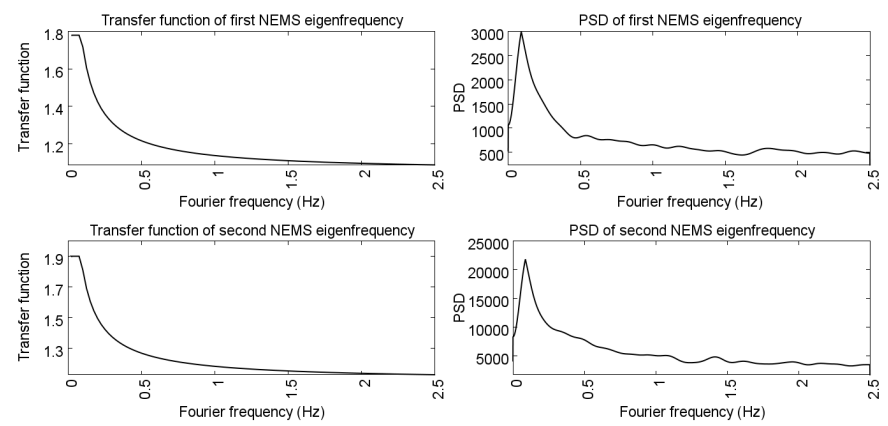

Figure 6: First line: Transfer function and PSD of generated pink noise for first NEMS resonance frequency trace. The resulting pink Gaussian noise has a mean equal to $0 \mathrm{~Hz}$ and a standard deviation equal to $45.36 \mathrm{~Hz}$. Second line : Transfer function and PSD of generated pink noise for second NEMS resonance frequency trace. The resulting pink Gaussian noise has a mean equal to $0 \mathrm{~Hz}$ and a standard deviation equal to $125.13 \mathrm{~Hz}$.

The main statistical features of the resulting mass distribution are given in table 2 for every method, and a view of the mass distribution is also given with Fig. 7. As we can see, the results are very similar to the case of white Gaussian noise. If the accuracy of our method is here slightly lower than the one of the reference method, it is still not of great importance according to the level reached. There is a $28 \%$ gain in resolution, which is of the same order of magnitude as the $37.8 \%$ found for the white Gaussian noise. This is visually confirmed by the mass histograms in Fig. 7 where the mass distribution looks sharper with our proposed method.

Figure 8 shows a comparison of the empirical cumulative distributions of our method and the reference method, for the case of pink noise. It is then clear 
that our proposed method produces a cumulative distribution closer in shape to the true cumulative distribution. This is further proof that our method better approximates the true underlying mass distribution than the reference method.

\begin{tabular}{|c|c|c|c|}
\hline & expected values & reference model & proposed model \\
\hline mean (MDa) & 106 & 105.96 & 105.95 \\
\hline std dev. (MDa) & $1.210^{-3}$ & 1.25 & 0.90 \\
\hline nb events & 210 & 211 & 210 \\
\hline
\end{tabular}

Table 2: Table of mass distribution statistics for exact case, reference method case and proposed method case for pink Gaussian noise describe in fig 6 .
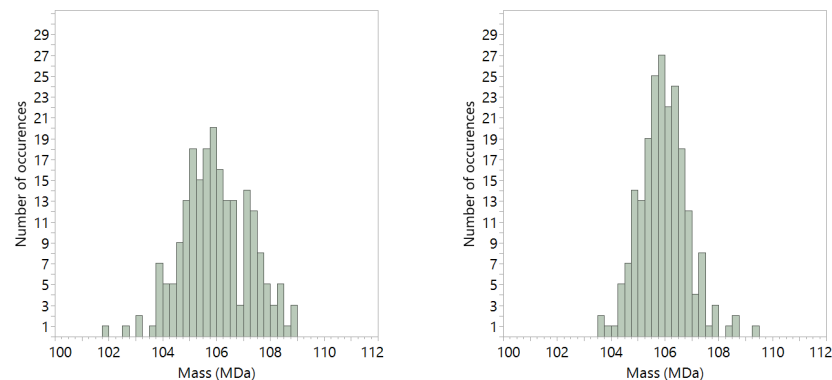

Figure 7: Case of pink Gaussian noise of means equal to $0 \mathrm{~Hz}$ and standard deviations equal to $45.36 \mathrm{~Hz}$ and $125.13 \mathrm{~Hz}$. Left: Histogram of mass distribution computed using the reference model ( 4 bins per mass unit). The mean of the resulting mass distribution is equal to 105.96 MDa and its standard deviation is equal to 1.25 MDa. The number of mass events in the range $[100,112]$ MDa is equal to 211. Right: Histogram of mass distribution computed using the proposed model ( 4 bins per mass unit). The mean of the resulting mass distribution is equal to $105.95 \mathrm{MDa}$ and its standard deviation is equal to 0.90 MDa. The number of mass events in the range $[100,112] \mathrm{MDa}$ is equal to 210. 


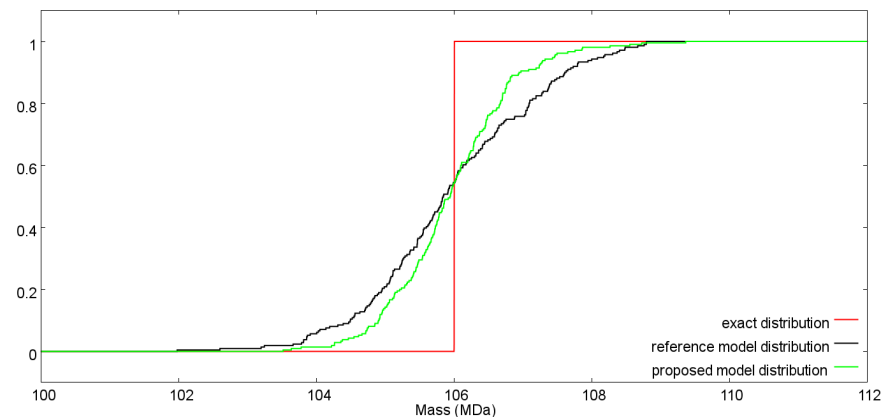

Figure 8: Case of pink Gaussian noise of means equal to $0 \mathrm{~Hz}$ and standard deviations equal to $45.36 \mathrm{~Hz}$ and $125.13 \mathrm{~Hz}$. Cumulative functions of mass distribution computed from exact distribution, reference method and proposed method.

We also considered pink noise for first and second NEMS resonance frequency traces which transfer functions and PSD are shown in Fig. 9
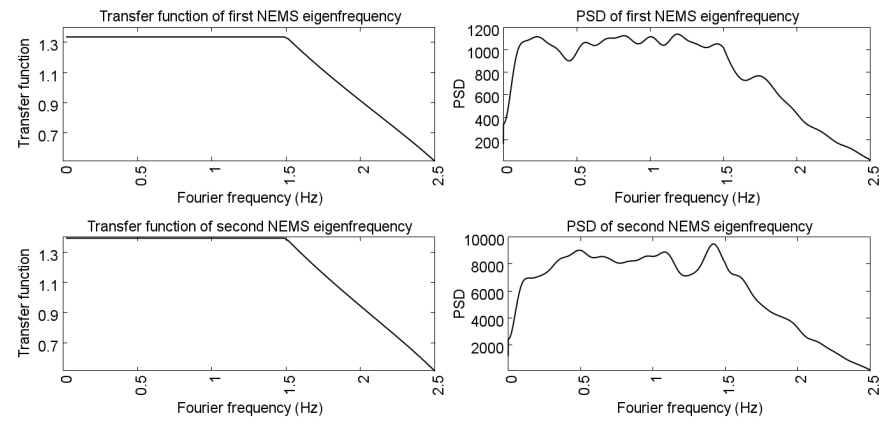

Figure 9: First line: Transfer function and PSD of generated pink noise for first NEMS resonance frequency trace. The resulting pink Gaussian noise has a mean equal to $0 \mathrm{~Hz}$ and a standard deviation equal to $45.22 \mathrm{~Hz}$. Second line : Transfer function and PSD of generated pink noise for second NEMS resonance frequency trace. The resulting pink Gaussian noise has a mean equal to $0 \mathrm{~Hz}$ and a standard deviation equal to $125.05 \mathrm{~Hz}$.

The noise means are both equal to $0 \mathrm{~Hz}$ and their standard deviations are equal to $45.22 \mathrm{~Hz}$ and $125.05 \mathrm{~Hz}$ respectively. As previously, noises energies are pretty similar to the ones described above. The SNR is equal to 1.0 for the first NEMS resonance frequency and is equal to 0.998 for the NEMS second resonance frequency. At last, note that the pink noise were generated from white noises of mean $0 \mathrm{~Hz}$ and standard deviations $28.94 \mathrm{~Hz}$ and $73.5 \mathrm{~Hz}$. As shown in table 3 , our method keeps its advantages: We have a gain of $24.1 \%$ in 
resolution, with only a $0.09 \%$ loss of accuracy.

\begin{tabular}{|c|c|c|c|}
\hline & expected values & reference model & proposed model \\
\hline mean (MDa) & 106 & 105.98 & 105.90 \\
\hline std dev. (MDa) & $1.210^{-3}$ & 1.33 & 1.01 \\
\hline nb events & 210 & 209 & 211 \\
\hline
\end{tabular}

Table 3: Table of mass distribution statistics for exact case, reference method case and proposed method case for pink Gaussian noise describe in fig 9

As in the previous pink noise test case, the empirical cumulative distribution we get with our denoising model is closer to the true cumulative distribution (see Fig. 11). It means that the true underlying mass distribution is better approximated with our method than with the reference one. Similarly, the cumulative distribution obtained with our method is sharper than with the reference method: It graphically confirms what was already shown in table 3 for standard deviations.
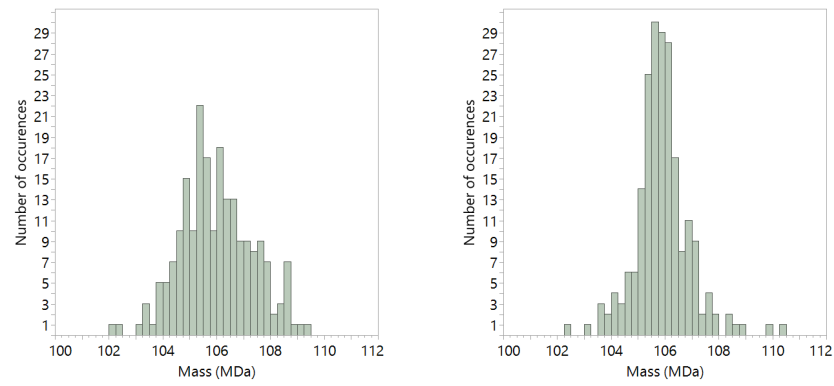

Figure 10: Case of pink Gaussian noise with means equal to $0 \mathrm{~Hz}$ and standard deviations equal to $45.22 \mathrm{~Hz}$ and $125.05 \mathrm{~Hz}$. Left: Histogram of mass distribution computed from reference model ( 4 bins per mass unit). The mean of the resulting mass distribution is equal to $105.98 \mathrm{MDa}$ and its standard deviation is equal to $1.33 \mathrm{MDa}$. The number of mass events in the range $[100,112] \mathrm{MDa}$ is equal to 209. Right: Histogram of mass distribution computed from proposed model ( 4 bins per mass unit). The mean of the resulting mass distribution is equal to $105.90 \mathrm{MDa}$ and its standard deviation is equal to 1.01 MDa. The number of mass events in the range $[100,112] \mathrm{MDa}$ is equal to 211. 


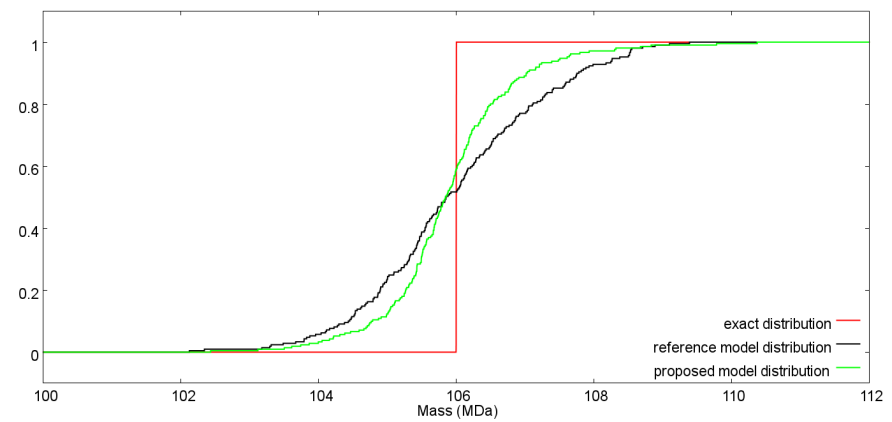

Figure 11: Case of pink Gaussian noise with means equal to $0 \mathrm{~Hz}$ and standard deviations equal to $45.22 \mathrm{~Hz}$ and $125.05 \mathrm{~Hz}$. Cumulative functions of mass distribution computed from exact distribution, reference method and proposed method.

\section{Conclusion}

The model presented in this report improves the denoising of NEMS signals for mass measurements by taking into account the NEMS physical properties. In particular, this model deals with the different types of noise affecting a NEMS and uses a single mathematical framework to treat them. An automatic computation of the model parameters is also proposed and we showed that these model parameters are directly linked to noise standard deviations, and thus have a simple physical meaning. We then upgraded our initial model to take into account the nonlinear coupling between NEMS signals. At last our specific numerical experiments showed a non-negligible gain in mass resolution compared to the standard method while keeping a good mass accuracy.

However we did not compare here the computational or time cost of our model with respect to the ones of the reference model. Clearly, we do not propose here an on-the-fly method: By essence, we require the whole NEMS signals to apply our denoising model and therefore, mass measurement experiments have to be completed to enable analysis. But in addition, the required computational effort is more important. Indeed, every step we described requires either solving a minimization problem or iterating on parameters to keep consistency with physics constraints whereas the reference method requires only a few elementary operations to work. However, the use of simple parallelization techniques can decrease the overall computation time of the proposed method, making it competitive compared to the reference method.

In daily experiments, the NEMS resonance frequency traces are also affected by other physical phenomena called drifts. These drifts would prevent us from using the proposed model in a straightforward way, as NEMS traces cannot be considered as constant piecewise functions over time. Capturing these drifts in a dedicated model could bring additional information on the NEMS physics 
or on the experimental conditions of a mass measurement. Hence, the issue of frequency drift is our next challenge, for which we will propose a new model in a future report.

\section{References}

[1] S. Dominguez-Medina, S. Fostner, M. Defoort, M. Sansa, A-K. Stark, M. Abdul Halim, E. Vernhes, M. Gely, G. Jourdan, T. Alava, P. Boulanger, C. Masselon, S. Hentz, "Neutral mass spectrometry of virus capsids above 100 megadaltons with nanomechanical resonators", in Science, vol. 362, no. 6417, pp. 918-922, 2018. [Online] Available: https://doi.org/10.1126/science.aat6457

[2] E. Sage, "New concept of mass spectrometer based on arrays of resonating nanostructure", PhD. dissertation, Université Grenoble Alpes, Grenoble, France, 2016. [Online] Available: https://tel.archives-ouvertes.fr/tel01178607

[3] R. Perenon, "Traitement de línformation en mode comptage appliqué aux détecteurs spectrométriques", Université Grenoble Alpes, Grenoble, France, 2013. [Online] Available: https://tel.archives-ouvertes.fr/tel00957594/document

[4] A. Langer, "Automated Parameter Selection for Total Variation Minimization in Image Restoration", in J. Math. Imaging Vis., no. 57, pp. 239-268, 2017. [Online] Available: https://doi.org/10.1007/s10851-016-0676-2

[5] A. Chambolle, "An algorithm for total variation minimization and applications", in J. Math. Imaging Vis., vol. 20, no. 1-2, pp. 89-97, 2004. [Online] Available: https://doi.org/10.1023/B:JMIV.0000011325.36760.1e

[6] A. Chambolle, P.-L. Lions, "Image recovery via total variation minimization and related problems", in Numerische Mathematik, no. 76, pp. 167-188, 1997. [Online] Available: https://doi.org/10.1007/s002110050258

[7] P. Weiss, L. Blanc-Féraud, G. Aubert, "Efficient Schemes for Total Variation Minimization Under Constraints in Image Processing", in SIAM Journal on Scientific Computing, vol. 31, no. 3, pp. 2047-2080, 2009. [Online] Available: https://doi.org/10.1137/070696143

[8] Y. Nesterov, "Lecture on Convex Optimization", 2 $2^{\text {nd }}$ ed., New York, N-Y, USA:Springer International Publishing, 2018. [Online] Available: https://doi.org/10.1007/978-3-319-91578-4

[9] A. Beck, M. Teboulle, "A Fast Iterative Shrinkage-Thresholding Algorithm for Linear Inverse Problems", in SIAM Journal on Imaging Sciences, vol. 2, no. 1, pp. 183-202, 2009. [Online] Available: https://doi.org/10.1137/080716542 
[10] L. I. Rudin, S. Osher, E. Fatemi, "Nonlinear total variation based noise removal algorithms", in Physica D: Nonlinear Phenomena, vol. 60, no. 1-4, pp. 259-268, 1992. [Online] Available: https://doi.org/10.1016/01672789(92)90242-F

[11] A. Bouchaala, A. H. Nayfeh, N. Jaber, M. I. Younis, "Mass and position determination in MEMS mass sensors: a theoretical and an experimental investigation", in Journal of Micromechanics and Microengineering, vol. 26, no. 10, pp. 105009, 2016. [Online] Available: https://doi.org/10.1088/0960$1317 / 26 / 10 / 105009$

[12] M. Sansa, E. Sage, E. C. Bullard, M. Gély, T. Alava, E. Colinet, A. K. Naik, L. G. Villanueva, L. Duraffourg, M. L. Roukes, G. Jourdan, S. Hentz, "Frequency fluctuations in silicon nanoresonators", in Nature nanotechnology, vol. 11, pp. 552-558, 2016. [Online] Available: https://doi.org/10.1038/nnano.2016.19

[13] R. H. Byrd, P. Lu, J. Nocedal, C. Zhu, "A limited memory algorithm for bound constrained optimization", in SIAM Journal on Scientific Computing, vol. 16, no. 5, pp. 1190-1208, 1995. [Online] Available: https://doi.org/10.1137/0916069

[14] C. Zhu, R. H. Byrd, P. Lu, J. Nocedal, "L-BFGS-B: Fortran subroutines for large-scale bound-constrained optimization", in ACM Transactions on Mathematical Software, vol. 23, no. 4, pp. 550-560, 1997. [Online] Available: https://doi.org/10.1145/279232.279236

[15] M. S. Hanay, S. Kelber, A. K. Naik, D. Chi, S. Hentz, E. C. Bullard, E. Colinet, L. Duraffourg, M. L. Roukes, "Single-protein nanomechanical mass spectrometry in real time", in Nature Nanotechnology, vol. 7, pp. 602-608, 2012. [Online] Available: https://doi.org/10.1038/nnano.2012.119 